Article

\title{
Effect of Lipid-Testing Interval on Stoke Risk among Newly Diagnosed Dyslipidemia Patients Initiated on Statins
}

\author{
Ahryoung Ko ${ }^{1,+} \mathbb{D}$, Seulggie Choi ${ }^{2,+}$, Jooyoung Chang ${ }^{2} \mathbb{D}$ and Sang Min Park ${ }^{1,2, *}$ \\ 1 Department of Family Medicine, Seoul National University Hospital, \\ Seoul National University College of Medicine, Seoul 03080, Korea; ahryoungko@gmail.com \\ 2 Department of Biomedical Sciences, Seoul National University Graduate School, Seoul 03082, Korea; \\ seulggie@gmail.com (S.C.); joomyjoo@gmail.com (J.C.) \\ * Correspondence: smpark.snuh@gmail.com; Tel.: +82-2-2072-3331; Fax: +82-2-766-3276 \\ + These authors contributed equally to this work.
}

Received: 25 March 2019; Accepted: 21 May 2019; Published: 24 May 2019

\begin{abstract}
Background: Although current guidelines recommend regular lipid testing for dyslipidemia patients, the effectiveness of regular lipid profile monitoring in clinical outcomes is unclear. (2) Methods: We assessed 64,664 newly diagnosed dyslipidemia patients from the Korean National Health Insurance Service Health Screening Cohort from 2003-2011 For lipid-testing frequency from all admission and outpatient records for 3 years after diagnosis. Participants were followed until 31 December 2015 for stroke. We used Cox regression analysis to determine the adjusted hazard ratio (aHR) for stroke according to lipid-testing interval. (3) Results: Compared to patients with lipid-testing intervals of $\leq 6$ months, patients with $>6$ to $\leq 12$ (aHR $1.32,95 \%$ confidence interval (CI) 1.08-1.61), >12 to $\leq 18$ (aHR 1.48, 95\% CI 1.20-1.82), and >18 (aHR 1.54, 95\% CI 1.25-1.90) month testing intervals had elevated risk of total stroke ( $p$ for trend $<0.001$ ). A significant association existed between lipid-testing interval and total and ischemic stroke risk in the $>6$ to $\leq 12$ (aHR 1.62, 95\% CI 1.19-2.21), >12 to $\leq 18$ (aHR 1.87, 95\% CI 1.36-2.58), and >18 (aHR 1.79, 95\% CI 1.30-2.48) month interval groups, but no significant association existed between lipid-testing interval and hemorrhagic stroke risk. (4) Conclusions: Lipid-testing intervals of more than 6 months may lead to increased stroke risk among newly diagnosed dyslipidemia patients after initiation of statin treatment. Lipid testing every 6 months can lower stroke risk among dyslipidemia patients.
\end{abstract}

Keywords: dyslipidemia; lipid-testing interval; stroke; statin

\section{Introduction}

Statin therapy has become the most important advancement in stroke prevention since the introduction of aspirin and blood pressure-lowering therapies. Statins not only lower the overall risk of stroke but also slow the progression of carotid atherosclerosis [1], reduce inflammation and endothelial dysfunction, decrease platelet aggregation to improve fibrinolysis, lower blood pressure, and decrease the risk of thromboembolic complications to the brain $[1,2]$.

There is robust evidence that lipid abnormalities such as high plasma triglycerides (TG), low-density lipoprotein cholesterol (LDL-C), and decreased high-density lipoprotein cholesterol (HDL-C) levels are associated with increased stroke risk [3,4]. Drugs used for lipid-lowering therapy, such as statins, reduce the level of LDL-C and triglycerides levels, and increase HDL-C through modulation of cholesterol ester transfer protein [5,6]. Regular lipid profile follow-up is needed to check for an adequate patient response to lipid-lowering therapy and evaluate whether dyslipidemia is well-managed $[7,8]$. 
Lipid measurements are essential for calculating an individual's risk of atherosclerotic cardiovascular disease (ASCVD), which includes stroke in addition to coronary heart disease and peripheral arterial disease, and determine when to initiate, adjust, or change lipid-lowering therapy [8,9]. Despite several clinical guidelines that recommend regular monitoring of lipid profiles to assess and improve adherence, the clinical benefit of such monitoring to detect non-adherence remains unproven. Recent lipid-management guidelines favor a risk-assessment approach that emphasizes lowering ASCVD risk rather than targeting specific LDL-C levels [9-11]. A limited number of studies have investigated the frequency of measuring lipid levels in patients who take lipid-lowering medications and its effects on long-term health such as risk of stroke, although stroke is one of the leading causes of death and disability worldwide [12].

We aimed to determine the effect of the lipid-test interval on the risk of developing strokes among newly diagnosed dyslipidemia patients initiated on statins. The patient population was obtained from the Korean National Health Insurance Service (NHIS) database.

\section{Subjects and Methods}

\subsection{Data Collection}

The study population was derived from the Korean NHIS database. In Korea, the NHIS provides universal healthcare to all Korean citizens, resulting in an enrollment rate of $97 \%$. The NHIS collects data from all hospital use including inpatient and outpatient visits, pharmaceutical drug prescriptions, and national health examinations [13]. For all enrollees aged 40 years or older, the NHIS provides biannual health screening examinations, which is comprised of a self-reported questionnaire on health behavior and medical history, measurements such as height, weight, and blood pressure, and blood tests. From this data, the NHIS constructed a cohort for research purposes called the National Health Insurance Service-National Health Screening Cohort (NHIS-HEALS), which contains information on health examinations, hospital use, and drug prescriptions among men and women aged 40 years or older at 2002 and were followed-up until 2015 [14]. NHIS-HEALS also contains information on the cause of death and death date, which was merged from the Statistics Korea database. The NHIS-HEALS database has previously been used for multiple epidemiological studies and its validity is described in detail elsewhere [14].

\subsection{Study Population}

After identifying newly diagnosed dyslipidemia patients during 2003-2011, the frequency of lipid testing was observed for the next 3 years after diagnosis date. Afterwards, participants were followed-up until stroke, death, or 31 December 2015, whichever came earliest. The index date was 3 years after diagnosis date of dyslipidemia. Among 75,944 newly diagnosed dyslipidemia patients during 2003-2011 who were not diagnosed with dyslipidemia in 2002, we excluded 2245 participants with missing values on covariates (Age $(n=26)$, Smoking $(n=1620)$, Alcohol $(n=184)$, Physical activity $(n=361)$, Body mass index $(n=9)$, Systolic blood pressure $(n=10)$, Fasting serum glucose $(n=16)$, Total cholesterol $(n=19))$. Furthermore, 8731 and 304 individuals who were diagnosed with cardiovascular disease or died before the index date were excluded, respectively. The study population consisted of 64,664 newly diagnosed dyslipidemia patients.

The Seoul National University Institutional Review Board (IRB) approved this study (IRB number: E-1803-046-928) and the requirement for informed consent was waived as the NHIS-HEALS database was constructed after anonymization according to strict confidentiality guidelines.

\subsection{Key Variables}

Dyslipidemia was defined when a participant was prescribed statin medication under the International Classification of Diseases, Tenth Revision (ICD-10) code pertaining to dyslipidemia (E78), along with at least one lipid blood test within 3 years of diagnosis. Lipid testing, which includes total 
cholesterol, TG, HDL-C, and LDL-C, was observed for all participants for 3 years after diagnosis of dyslipidemia. Lipid-testing interval was defined as the average interval between lipid testing during the observed 3-year span. The 3-year interval was divided into 6 months, resulting in a total of 6 half-year intervals, after which whether or not each individual had undergone lipid testing for each interval was determined. Then, the total number of intervals was divided by the cumulative number of lipid tests, resulting in the average lipid-testing interval. The study population was divided according to the average lipid-testing interval of $\leq 6,6$ to 12,12 to 18 , and $\geq 18$ months.

\subsection{Identification of Stoke}

Stroke was defined as 2 or more days of hospitalization or death with the cause of death under ICD-10 codes pertaining to total stroke (I60-I69). The ICD-10 codes are in line with those used by the American Heart Association [15] Total stroke was further divided into ischemic stroke (ICD-10 code I63) and hemorrhagic stroke (ICD-10 codes I61 and I62).

\subsection{Statistical Analysis}

For determining the differences in descriptive characteristics according to average lipid-testing interval groups, Chi squared test was used for categorical variables and analysis of variance for continuous variables (Table 1). Cox proportional hazards regression was used obtain the adjusted hazard ratios (aHRs) and 95\% confidence intervals (CIs) for stroke risk according to average lipid-testing interval.

Cox regression and competing-risks survival regression based on Fine and Gray's model, were conducted to estimate the hazard ratios of stroke (Table 2). Also, the risk of coronary heart disease and cardiovascular disease mortality risk was determined according to lipid-testing frequency.

The assumption of proportionality for the Cox regression analysis was graphically tested and verified using the Schoenfeld residual method. The considered covariates include age (continuous, years), sex (categorical, men and women), household income (categorical, 1st, 2nd, 3rd, and 4th quartiles), smoking (categorical, never, past, and current smokers), alcohol consumption (categorical, none, $<1,1-2,3-4$, and $\geq 5$ times per week), physical activity (categorical, none, 1-2, 3-4, 5-6, and 7 times per week), body mass index (continuous, $\mathrm{kg} / \mathrm{m}^{2}$ ), systolic blood pressure (continuous, $\mathrm{mmHg}$ ), fasting serum glucose (continuous, $\mathrm{mg} / \mathrm{dL}$ ), total cholesterol (continuous, $\mathrm{mg} / \mathrm{dL}$ ), Charlson comorbidity index (continuous), statin adherence (continuous, medication possession ratio), outpatient department visits (OPD) (categorical, 1st, 2nd, 3rd, and 4th quartiles), and enrollment year (continuous, year). Household income was determined by the insurance premium and body mass index by dividing the weight in kilograms by height in meters squared. Statin adherence was measured by medication possession ratio (MPR), which is calculated by dividing the defined daily dose (DDD) between the first and last prescription or to the end of follow-up during the 3 years after diagnosis of dyslipidemia. Statin dosage, standardized by DDD, was calculated according to the Anatomical Therapeutic Chemical classification system of drugs by the World Health Organization Collaborating Center for Drug Statistics Methodology [16]. Enrollment year was defined as the year of dyslipidemia diagnosis. Stratified analyses for total stroke risk according to subgroups of age, smoking, physical activity, alcohol consumption, total cholesterol, body mass index, outpatient department visits, and statin adherence were conducted.

Statistical significance was defined as a $p$ value of less than 0.05 in a 2-sided manner. All data collection and statistical analyses were conducted using SAS 9.4 (SAS Institute Inc, Cary, NC, USA). 
Table 1. Descriptive characteristics of the study population.

\begin{tabular}{|c|c|c|c|c|c|}
\hline & \multicolumn{4}{|c|}{ Average Lipid-Testing Interval, Months } & \multirow[b]{2}{*}{$p$ Value } \\
\hline & $\leq 6$ & 6 to 12 & 12 to 18 & $\geq 18$ & \\
\hline Number of people & 4470 & 28,189 & 15,727 & 16,278 & \\
\hline Age, years, mean (SD) & $60.2(8.1)$ & $60.4(8.2)$ & $60.6(8.5)$ & $60.2(8.5)$ & $<0.001$ \\
\hline \multicolumn{6}{|l|}{ Sex, N (\%) } \\
\hline Men & $2046(45.8)$ & $11,233(39.9)$ & $6596(41.9)$ & $7356(45.2)$ & \multirow[t]{2}{*}{$<0.001$} \\
\hline Women & $2424(54.2)$ & $16,956(60.2)$ & $9131(58.1)$ & $8922(54.8)$ & \\
\hline \multicolumn{6}{|l|}{ Household income, quartiles, N (\%) } \\
\hline 1st (highest) & $1872(41.9)$ & $10,044(35.6)$ & $5433(34.6)$ & $5552(34.1)$ & \multirow[t]{4}{*}{$<0.001$} \\
\hline 2nd & $1260(28.2)$ & 8379 (29.7) & 4726 (30.1) & 4887 (30.0) & \\
\hline 3 rd & 800 (17.9) & 5525 (19.6) & 3241 (20.6) & 3369 (20.7) & \\
\hline 4th (lowest) & $538(12.0)$ & $4241(15.0)$ & $2327(14.8)$ & $2470(15.2)$ & \\
\hline \multicolumn{6}{|l|}{ Smoking status, N (\%) } \\
\hline Never smoker & $3245(72.6)$ & $21,209(75.2)$ & $11,601(73.8)$ & $11,570(71.1)$ & \multirow[t]{3}{*}{$<0.001$} \\
\hline Past smoker & 787 (17.6) & 3917 (13.9) & 2167 (13.8) & 2317 (14.2) & \\
\hline Current smoker & $438(9.8)$ & $3063(10.9)$ & $1959(12.5)$ & $2391(14.7)$ & \\
\hline \multicolumn{6}{|l|}{ Physical activity, times per week, N (\%) } \\
\hline None & $2090(46.8)$ & $14,089(50.0)$ & $8161(51.9)$ & $8499(52.2)$ & \multirow[t]{5}{*}{$<0.001$} \\
\hline $1-2$ & 1054 (23.6) & $6480(23.0)$ & 3627 (23.1) & 3805 (23.4) & \\
\hline $3-4$ & $709(15.9)$ & $4177(14.8)$ & $2201(14.0)$ & $2235(13.7)$ & \\
\hline $5-6$ & $322(7.2)$ & $1730(6.1)$ & $877(5.6)$ & $891(5.5)$ & \\
\hline 7 & $295(6.6)$ & $1713(6.1)$ & $861(5.5)$ & $848(5.2)$ & \\
\hline \multicolumn{6}{|l|}{$\begin{array}{c}\text { Alcohol consumption, times per week, } \mathrm{N} \\
(\%)\end{array}$} \\
\hline None & $3052(68.3)$ & $19,069(67.7)$ & $10,353(65.8)$ & $10,246(62.9)$ & \multirow[t]{5}{*}{$<0.001$} \\
\hline$<1$ & $616(13.8)$ & $3878(12.8)$ & $2157(13.7)$ & $2506(15.4)$ & \\
\hline $1-2$ & $419(9.4)$ & $2701(9.6)$ & $1624(10.3)$ & $1750(10.8)$ & \\
\hline $3-4$ & $259(5.8)$ & $1731(6.1)$ & $1003(6.4)$ & $1189(7.3)$ & \\
\hline$\geq 5$ & $124(2.8)$ & $810(2.9)$ & $590(3.8)$ & $587(3.6)$ & \\
\hline Body mass index, $\mathrm{kg} / \mathrm{m}^{2}$, mean (SD) & $24.7(3.0)$ & $24.8(3.0)$ & $24.7(2.9)$ & $24.6(2.9)$ & $<0.001$ \\
\hline Systolic blood pressure, $\mathrm{mmHg}$, mean (SD) & $126.1(15.4)$ & $126.8(15.1)$ & $127.2(15.2)$ & $126.9(15.1)$ & $<0.001$ \\
\hline Fasting serum glucose, mg/dL, mean (SD) & $108.0(30.1)$ & $105.5(29.3)$ & $104.0(28.0)$ & $103.1(27.8)$ & $<0.001$ \\
\hline Total cholesterol, mg/dL, mean (SD) & $189.3(44.8)$ & $202.7(45.8)$ & $209.4(45.9)$ & $214.6(44.6)$ & $<0.001$ \\
\hline \multicolumn{6}{|l|}{ OPD visits, average interval, N (\%) } \\
\hline 1st (mean 9 visits per year) & $677(15.2)$ & $5008(17.8)$ & $4237(26.9)$ & $5989(36.8)$ & \multirow[t]{4}{*}{$<0.001$} \\
\hline 2nd (mean 17 visits per year) & $1151(25.8)$ & 7368 (26.1) & 4079 (25.9) & $4408(24.9)$ & \\
\hline 3rd (mean 26 visits per year) & $1242(27.8)$ & $7519(26.7)$ & $3711(23.6)$ & $3342(20.5)$ & \\
\hline 4th (mean 53 visits per year) & $1400(31.3)$ & $8294(29.4)$ & $3700(23.5)$ & $2899(17.8)$ & \\
\hline Statin adherence, MPR, mean (SD) & $0.6(0.3)$ & $0.5(0.3)$ & $0.4(0.3)$ & $0.3(0.3)$ & $<0.001$ \\
\hline \multicolumn{6}{|l|}{ Charlson comorbidity index, N (\%) } \\
\hline 0 & $310(6.9)$ & $2502(8.9)$ & $1801(11.5)$ & $2371(14.6)$ & \multirow[t]{4}{*}{$<0.001$} \\
\hline 1 & 731 (16.4) & $5501(19.5)$ & $3683(23.4)$ & $4469(27.5)$ & \\
\hline 2 & $938(21.0)$ & $6789(24.1)$ & 3979 (25.3) & $4137(25.4)$ & \\
\hline$\geq 3$ & $2491(55.7)$ & $13,397(47.5)$ & $6264(39.8)$ & $5301(32.6)$ & \\
\hline
\end{tabular}

$p$ value calculated by the Chi squared test for categorical variables and analysis of variance for continuous variables. Acronyms: SD, standard deviation; OPD, outpatient department; MPR, medication possession ratio. 
Table 2. Hazard ratios for stroke according to average lipid-testing interval among newly diagnosed dyslipidemia patients.

\begin{tabular}{|c|c|c|c|c|c|}
\hline & \multicolumn{4}{|c|}{ Average Lipid-Testing Interval, Months } & \multirow[b]{2}{*}{$p$ for Trend } \\
\hline & $\leq 6$ & 6 to 12 & 12 to 18 & $\geq 18$ & \\
\hline \multicolumn{6}{|l|}{ Total stroke } \\
\hline Events & 111 & 885 & 536 & 546 & \\
\hline Person-years & 21,458 & 133,245 & 73,079 & 75,609 & \\
\hline $\operatorname{aHR}(95 \% \mathrm{CI})$ & 1.00 (reference) & $1.32(1.08-1.61)$ & $1.48(1.20-1.82)$ & $1.54(1.25-1.90)$ & $<0.001$ \\
\hline \multicolumn{6}{|l|}{ Ischemic stroke } \\
\hline Events & 45 & 451 & 287 & 269 & \\
\hline Person-years & 21,458 & 133,245 & 73,079 & 75,609 & \\
\hline aHR $(95 \%$ CI $)$ & 1.00 (reference) & $1.62(1.19-2.21)$ & $1.87(1.36-2.58)$ & $1.79(1.30-2.48)$ & 0.004 \\
\hline \multicolumn{6}{|c|}{ Hemorrhagic stroke } \\
\hline Events & 22 & 77 & 49 & 56 & \\
\hline Person-years & 21,458 & 133,245 & 73,079 & 75,609 & \\
\hline aHR $(95 \% \mathrm{CI})$ & 1.00 (reference) & $0.61(0.38-1.00)$ & $0.72(0.43-1.21)$ & $0.85(0.50-1.42)$ & 0.455 \\
\hline
\end{tabular}

Hazard ratio calculated by Cox proportional hazards regression after adjustments for age, sex, household income, smoking status, physical activity, alcohol consumption, body mass index, systolic blood pressure, fasting serum glucose, total cholesterol, outpatient department visits, statin adherence, Charlson comorbidity index, and enrollment year. Acronyms: aHR, adjusted hazard ratio; $\mathrm{CI}$, confidence interval.

\section{Results}

Table 1 depicts the descriptive characteristics of the study population. The number of participants with average lipid-testing intervals of $\leq 6,6$ to 12,12 to 18 , and $\geq 18$ months are 4470, 28,189, 15,727, and 16,278, respectively. The mean (standard deviation, SD) age for participants with average lipid-testing intervals of $\leq 6,6$ to 12,12 to 18 , and $\geq 18$ months are 60.2 (8.1), 60.4 (8.2), 60.6 (8.5), and 60.2 (8.5) years, respectively. Compared to those with average lipid-testing interval of $\leq 6$ months, those with an average lipid-testing interval of $\geq 18$ months tended to have lower household income, be current smokers, exercise less, consume less alcohol, visit the outpatient department less frequently, and have less comorbid conditions (all $p<0.001$ ).

Results from the effect of lipid-testing interval on stroke risk are shown in Table 2. Compared to those with lipid-testing interval of $\leq 6$ months, participants with 6 to 12 (aHR 1.32, 95\% CI 1.08-1.61), 12 to 18 (aHR 1.48, 95\% CI 1.20-1.82), and $\geq 18$ months (aHR 1.54, 95\% CI 1.25-1.90) had elevated risk of total strokes. Participants with lipid-testing interval of 6 to 12 (aHR 1.62, 95\% CI 1.19-2.21), 12 to 18 (aHR 1.87, 95\% CI 1.36-2.58), and $\geq 18$ months (aHR 1.79, 95\% CI 1.30-2.48) had elevated risk of ischemic stroke compared to those with lipid-testing interval of $\leq 6$ months. No significant association was found between lipid-testing interval and hemorrhagic stroke risk (6 to 12 (aHR 0.61 , 95\% CI 0.38-1.00), 12 to 18 (aHR 0.72, 95\% CI 0.43-1.21), and $\geq 18$ months (aHR 0.85, 95\% CI 0.50-1.42).

Tables 3 and A1 depict the results from stratified analyses on the effect of average lipid-testing interval on total strokes, respectively. The positive association between lipid-testing interval and total strokes was preserved among subgroups of sex, drug adherence and outpatient department visits (Table 3). The increased risk effect for stroke with longer lipid-testing intervals tended to be more prevalent among those who were within aged $\geq 60$ years (Table $3, p<0.05$ ), past or current smokers, exercised, did not consume alcohol, total cholesterol $<240 \mathrm{mg} / \mathrm{dL}$ and had body mass index $<25 \mathrm{~kg} / \mathrm{m}^{2}$ (Table A1, all $p<0.05$ ). Finally, the risk of coronary heart disease or cardiovascular disease mortality was not increased upon greater lipid-testing intervals (Table A2). 
Table 3. Stratified analysis on the effect of average lipid-testing interval on total stroke among newly diagnosed dyslipidemia patients according to subgroups of age, sex, outpatient department visits, and statin adherence.

\begin{tabular}{cccccc}
\hline \multicolumn{5}{c}{ Adjusted Hazard Ratio (95\% Confidence Interval) } & \\
\cline { 2 - 5 } & \multicolumn{5}{c}{ Average Lipid-Testing Interval, Months } \\
\hline Age & $\mathbf{6}$ to 12 & $\mathbf{1 2}$ to 18 & $\geq \mathbf{1 8}$ & p for Trend \\
\hline <0 years & 1.00 (reference) & $1.19(0.85-1.67)$ & $1.41(0.99-2.02)$ & $1.36(0.94-1.96)$ & 0.050 \\
$\geq 60$ years & 1.00 (reference) & $1.43(1.12-1.83)$ & $1.64(1.27-2.12)$ & $1.81(1.40-2.34)$ & $<0.001$ \\
\hline Sex & & & & \\
Men & 1.00 (reference) & $1.36(1.00-1.84)$ & $1.51(1.10-2.08)$ & $1.59(1.16-2.19)$ & 0.004 \\
Women & 1.00 (reference) & $1.28(0.99-1.67)$ & $1.44(1.09-1.90)$ & $1.50(1.13-1.98)$ & 0.003 \\
\hline OPD visits & & & & \\
Upper half & 1.00 (reference) & $1.09(0.76-1.55)$ & $1.12(0.78-1.60)$ & $1.28(0.92-1.79)$ & 0.031 \\
Lower half & 1.00 (reference) & $1.17(0.91-1.49)$ & $1.17(0.91-1.52)$ & $1.29(1.01-1.64)$ & 0.040 \\
\hline Statin adherence & & & & & \\
MPR $<0.5$ & 1.00 (reference) & $1.09(0.84-1.42)$ & $1.04(0.79-1.36)$ & $1.23(0.96-1.59)$ & 0.041 \\
MPR $\geq 0.5$ & 1.00 (reference) & $1.21(0.88-1.66)$ & $1.34(0.97-1.85)$ & $1.39(1.03-1.89)$ & 0.022 \\
\hline
\end{tabular}

Hazard ratio calculated by Cox proportional hazards regression after adjustments for age, sex, household income, smoking status, physical activity, alcohol consumption, body mass index, systolic blood pressure, fasting serum glucose, total cholesterol, outpatient department visits, statin adherence, Charlson comorbidity index, and enrollment year. Acronyms: OPD, outpatient department; MPR, medication possession ratio.

\section{Discussion}

In this retrospective cohort study, we showed that an association existed between increased lipid-testing intervals of more than 6 months and elevated risk of total stroke among newly diagnosed dyslipidemia patients initiated on statins. Compared to those with lipids tested at $<6$ months intervals, those patients tested every 6-12 months, 12-18 months and $>18$ months had higher rates of ischemic stroke event rates over the next three 6-month intervals compared to event rates in the first 6 months. There was no significant increase in hemorrhagic strokes, but when we combined the stroke diagnoses, there was a significant combined increase over 6 months with less average lipid screening. To the best of our knowledge, this was the first study to show an elevated the risk of stroke when the interval of lipid testing was longer than 6 months among patients who initiated statin treatment.

There is insufficient evidence on the stroke risk of those who do not undergo regular lipid testing after initiating lipid-lowering medications, despite the high prevalence of lipid testing in the clinical practice [17]. Lipid-lowering management with statins and lipid testing is important to improve or optimize the clinical status of patients. A previous study has suggested that because the true underlying cholesterol levels change slowly, clinicians may monitor cholesterol too often to evaluate real trends of lipid profiles [18]. Guidelines recommend that clinicians monitor patients' cholesterol levels to assess adherence to lipid-lowering medications or assess the risk of ASCVD by reviewing their lipid profiles $[9,19,20]$. For patients, blood cholesterol measurements may provide motivation to adhere to lipid profile management, including adherence to medications and modifications of lifestyles [21]. In this study, we have shown an association between longer lipid-testing intervals of more than 6 months and elevated risk of stroke, even after adjustments for a wide range of potential confounders such as drug adherence, OPD visits, and health behaviors that could impact lipid modifications.

Previous studies suggested that frequent and regular lipid monitoring played an important role in long-term adherence [22,23]. Short-term follow-up with lipid testing after initiation of statins might be beneficial in cases of poor compliance to therapy. Patients who visit hospitals frequently are more likely to have the chance to consult with their physicians. Thus, OPD visits may act as a surrogate marker for a chance for a therapeutic intervention through physician follow-up. Management by physicians is crucial for optimizing adherence to medications and health behaviors that include smoking habit, 
regular exercise, diet, and alcohol consumption [24]. Therefore, we conducted stratified analyses in this study according to the subgroups of drug adherence and OPD visits. Several mechanisms might explain the risk-increasing effect for stroke according to the increasing lipid-testing intervals in our study. First, the lack of lipid testing might lead to inappropriate adjustment of lipid-lowering therapy because the lipid profiles are unknown. Possibly, a patient has a high drug adherence without monitoring laboratory results and, lipid management care might be suboptimal because the patient could be undertreated but a less intensive lipid management and would not achieve target LDL-C levels. Second, lack of awareness of lipid levels might lead to inappropriate risk-assessment and lifestyle intervention. A physician would need to control the ASCVD risk of a patient according to the result of the lipid laboratory test. If the test is not conducted, a physician would have less chance to educate, emphasize, or modify the patient's health behaviors. Modifiable risk factors of stroke such as elevated blood pressure, serum glucose, cholesterol levels and obesity could be prevented and controlled by offering lifestyle changes or explaining the benefits of a healthy lifestyle to dyslipidemia patients $[25,26]$. Third, knowledge of the cholesterol level may be important to motivate patients to talk with physicians and change behaviors to improve their cholesterol levels. Even though a randomized trial of the motivational effect of cholesterol measurement in general practice has shown only negligible benefit [27], a recent trial of diabetes patients randomized to self-monitoring their risk factors showed better achievement of target measurements of blood pressure, LDL-C, and $\mathrm{HbA1c}$, as well as a reduction in clinical events [21]. Other studies of type 2 diabetes and hypertension patients have showed that well-informed and motivated patients were more insistent to reach and maintain target values and, ultimately reduce the risk of stroke [28,29]. Likewise, knowing their cholesterol levels would motivate and encourage patients to comply with lipid-lowering therapy to improve their dyslipidemia and cardiovascular health.

As pointed out by other well-conducted studies, besides the anti-stroke effect of lipid-lowering agents, multi-factor risk factors should also be taken into consideration including stroke subtype [30,31]. Only the risk of ischemic stroke increased with increasing lipid-test interval, but the effect for the risk of hemorrhagic stroke was not evident in our study. First, our expectation from previous studies is that the incidence of obstructive vascular disease is proportional to the normal LDL-C concentration. In the Multiple Risk Factor Intervention Trial (MRFIT), which investigated the association between cholesterol and other underlying pathologic types of strokes and The Copenhagen City Heart Study (CCHS) had a similar dose-response relationship between serum cholesterol and ischemic stroke risk, but the risk of cholesterol and hemorrhagic stroke was reversed [32,33]. Second, our results can be expected from the result that statins reduce ischemic stroke and do not affect cerebral hemorrhage. Many recent studies have focused on the effect of statins on the development of stroke subtypes, and reported reductions in the risk of ischemic stroke and a significant increase in the risk of hemorrhagic stroke [34]. Therefore, the results of this study have suggested that intensive lipid profile management and correction of risk factors for stroke are necessary to improve clinical outcome. Careful consideration is needed when interpreting the exact reason for the inconsistency of ischemic stroke and bleeding risk according to the interval of lipid testing, and should be investigated further.

Several limitations must be considered when interpreting the results from our study. First, there could be an inaccuracy of the information in defining newly diagnosed dyslipidemia patients due to the nature of the claims database. However, previous studies for the diagnosis codes of the NHIS data have shown that $70 \%$ of the data from the NHIS database matched with those from patients' medical records $[35,36]$. Second, we could not account for LDL -C levels due to the lack of information-not only the test for LDL-C, but also total cholesterol, TG and HDL-C levels not included in this study. Although high LDL-C levels are necessary to prescribe lipid-lowering medications [37], total cholesterol, plasma TG level, and HDL-C level have previously been shown to be associated with several adverse outcomes [38-40]. Regarding the review of lipid abnormality with elevated total cholesterol, plasma TG, and decreased HDL-C concentrations, our study investigated the effect of monitoring lipid profiles in patients initiating statins. Third, we could not determine if dyslipidemia 
patients who underwent undergone stable lipid therapy or achieved treatment goals after initiating statins was due to the lack of lipid levels. Therefore, future studies that differentiate patients should be conducted to determine the effect of the testing interval on stroke event between the two groups of before and after the achieved treatment goal [41]. Fourth, we did not consider the effect of lipid monitoring on stroke risk beyond 3 years after initial diagnosis. The early pattern of lipid monitoring might represent the patients' general tendencies. These results also emphasized the importance of early frequent follow-up with lipid testing. Finally, our results given in the manuscript showing that lipid-testing frequency was not associated with higher risk of coronary heart disease or cardiovascular disease mortality. These results imply that intensive lipid profile management and modification of major risk factor for strokes is required for improving clinical outcome. On the other hand, CHD risk may have sufficiently decreased by taking statin medications, resulting in a weaker protective effect for CHD upon frequent lipid testing. It remains cautious in interpreting our results the exact reasons for the discrepancy in total stroke and CHD risk according to lipid-testing interval and merit further investigation.

The association of lipid-testing interval with coronary artery disease or CVD mortality, however, could not be assessed properly and thus merit future studies

\section{Conclusions}

This is the first study of a relatively large population to report the elevated risk of stroke according to longer lipid-testing intervals among patients who initiated statins therapy. These results suggest that longer lipid-testing intervals of more than 6 months might lead to elevated risk of stroke. Dyslipidemia patients who begun statins should be monitored for lipid levels to benefit from reduced risk of ASCVD. Our findings have also supported the 6 months interval for lipid testing among newly diagnosed dyslipidemia patients who initiated lipid-lowering drugs suggested by current guidelines for management [10]. This study also provides the grounds for physicians to assess appropriate ASCVD risk and lifestyle intervention to dyslipidemia patients and emphasize the need for strict dyslipidemia control.

Author Contributions: Conceptualization, A.K., S.C.; Data curation, S.C. and J.C.; Formal analysis, S.C.; Methodology, A.K., S.C., J.C. and S.M.P.; Supervision, S.M.P.; Writing—original draft, A.K., S.C.; Writing—review \& editing, S.C., J.C. and S.M.P.

Funding: This research was supported by the SNUH research fund (grant No. 04-2018-0370) and the Basic Science Research Program through the National Research Foundation of Korea (NRF) funded by the Ministry of Education (grant number 2017R1D1A1B03033721).

Acknowledgments: The authors thank the other participants of the study for their valuable contributions. We would like to thank the National Health Insurance Service for providing the database for research purposes (NHIS-2015-1-074).

Conflicts of Interest: S.C. received a scholarship from the BK21-plus education program provided by the National Research Foundation of Korea. No other conflicts are reported.

\section{Appendix A}

Table A1. Stratified analysis on the effect of average lipid-testing interval on total stroke among newly diagnosed dyslipidemia patients according to subgroups of smoking status, physical activity, alcohol consumption, total cholesterol, body mass index.

\begin{tabular}{cccccc}
\hline & \multicolumn{5}{c}{ Adjusted Hazard Ratio (95\% Confidence Interval) } \\
\cline { 2 - 5 } & \multicolumn{5}{c}{ Average Lipid-Testing Interval, Months } \\
\hline Smoking status & $\mathbf{6}$ & $\mathbf{6}$ to $\mathbf{1 2}$ & $\mathbf{1 2}$ to $\mathbf{1 8}$ & $\mathbf{1 1 8}$ & $\boldsymbol{p}$ for Trend \\
\hline Never smoker & 1.00 (reference) & $1.25(0.99-1.58)$ & $1.12(0.88-1.43)$ & $1.31(1.05-1.65)$ & 0.061 \\
Past or current smoker & 1.00 (reference) & $0.85(0.57-1.28)$ & $1.30(0.87-1.95)$ & $1.32(0.91-1.93)$ & 0.002 \\
\hline
\end{tabular}


Table A1. Cont.

\begin{tabular}{|c|c|c|c|c|c|}
\hline & \multicolumn{4}{|c|}{ Adjusted Hazard Ratio (95\% Confidence Interval) } & \multirow[b]{3}{*}{$p$ for Trend } \\
\hline & \multicolumn{4}{|c|}{ Average Lipid-Testing Interval, Months } & \\
\hline & $\leq 6$ & 6 to 12 & 12 to 18 & $\geq \mathbf{1 8}$ & \\
\hline \multicolumn{6}{|c|}{ Physical activity } \\
\hline No & 1.00 (reference) & $1.20(0.91-1.59)$ & $1.15(0.86-1.54)$ & $1.29(0.98-1.69)$ & 0.094 \\
\hline Yes & 1.00 (reference) & $1.08(0.81-1.44)$ & $1.17(0.87-1.58)$ & $1.34(1.01-1.76)$ & 0.005 \\
\hline \multicolumn{6}{|c|}{ Alcohol consumption } \\
\hline No & 1.00 (reference) & $1.21(0.95-1.54)$ & $1.19(0.93-1.53)$ & $1.37(1.08-1.73)$ & 0.006 \\
\hline Yes & 1.00 (reference) & $0.99(0.68-1.43)$ & $1.09(0.74-1.59)$ & $1.15(0.81-1.64)$ & 0.158 \\
\hline \multicolumn{6}{|l|}{ Total cholesterol } \\
\hline$<240 \mathrm{mg} / \mathrm{dL}$ & 1.00 (reference) & $1.10(0.88-1.36)$ & $1.11(0.88-1.39)$ & $1.30(1.05-1.60)$ & 0.002 \\
\hline$\geq 240 \mathrm{mg} / \mathrm{dL}$ & 1.00 (reference) & $1.34(0.78-2.30)$ & $1.37(0.79-2.38)$ & $1.35(0.80-2.29)$ & 0.557 \\
\hline \multicolumn{6}{|c|}{ Body mass index } \\
\hline$<25 \mathrm{~kg} / \mathrm{m}^{2}$ & 1.00 (reference) & $1.21(0.91-1.60)$ & $1.28(0.96-1.70)$ & $1.48(1.13-1.93)$ & $<0.001$ \\
\hline$\geq 25 \mathrm{~kg} / \mathrm{m}^{2}$ & 1.00 (reference) & $1.06(0.79-1.41)$ & $1.02(0.75-1.38)$ & $1.11(0.84-1.47)$ & 0.416 \\
\hline
\end{tabular}

Hazard ratio calculated by Cox proportional hazards regression after adjustments for age, sex, household income, smoking status, physical activity, alcohol consumption, body mass index, systolic blood pressure, fasting serum glucose, total cholesterol, outpatient department visits, statin adherence, Charlson comorbidity index, and enrollment year.

Table A2. Hazard ratios for cardiovascular mortality and coronary artery disease according to average lipid-testing interval among newly diagnosed dyslipidemia patients.

\begin{tabular}{|c|c|c|c|c|c|}
\hline & \multicolumn{4}{|c|}{ Average Lipid-Testing Interval, Months } & \multirow[b]{2}{*}{$p$ for Trend } \\
\hline & $\leq 6$ & 6 to 12 & 12 to 18 & $\geq 18$ & \\
\hline \multicolumn{6}{|c|}{ Cardiovascular mortality } \\
\hline Events & 11 & 106 & 70 & 59 & \\
\hline Person-years & 21,458 & 133,245 & 73,079 & 75,609 & \\
\hline aHR $(95 \%$ CI $)$ & 1.00 (reference) & $1.52(0.82-2.84)$ & $1.79(0.94-3.41)$ & $1.58(0.82-3.06)$ & 0.306 \\
\hline \multicolumn{6}{|c|}{ Coronary heart disease } \\
\hline Events & 167 & 1046 & 591 & 511 & \\
\hline Person-years & 21,458 & 133,245 & 73,079 & 75,609 & \\
\hline aHR $(95 \% \mathrm{CI})$ & 1.00 (reference) & $1.09(0.92-1.28)$ & $1.19(1.00-1.42)$ & $1.05(0.87-1.25)$ & 0.780 \\
\hline
\end{tabular}

Hazard ratio calculated by Cox proportional hazards regression after adjustments for age, sex, household income, smoking status, physical activity, alcohol consumption, body mass index, systolic blood pressure, fasting serum glucose, total cholesterol, outpatient department visits, statin adherence, Charlson comorbidity index, and enrollment year. Acronyms: aHR, adjusted hazard ratio; $\mathrm{CI}$, confidence interval.

\section{References}

1. Amarenco, P.; Lavallée, P.; Touboul, P.-J. Stroke prevention, blood cholesterol, and statins. Lancet Neurol. 2004, 3, 271-278. [CrossRef]

2. Levine, G.N.; Keaney, J.F., Jr.; Vita, J.A. Cholesterol reduction in cardiovascular disease-Clinical benefits and possible mechanisms. N. Engl. J. Med. 1995, 332, 512-521. [CrossRef] [PubMed]

3. Rifkind, B. The lipid research clinics coronary primary prevention trial results. I. Reduction in incidence of coronary heart disease. J. Am. Med. Assoc. 1984, 251, 351-364.

4. Manninen, V. Lipid alterations and decline in the incidence of coronary heart disease in the Helsinki Heart Study. JAMA 1988, 260, 641-651. [CrossRef] [PubMed]

5. Stein, E.A.; Lane, M.; Laskarzewski, P. Comparison of Statins in Hypertriglyceridemia. Am. J. Cardiol. 1998, 81, 66B-69B. [CrossRef]

6. Schaefer, E.J.; Wolfe, M.L.; Bloedon, L.T.; Digenio, A.G.; Clark, R.W.; Mancuso, J.P.; Brousseau, M.E.; Rader, D.J. Effects of an Inhibitor of Cholesteryl Ester Transfer Protein on HDL Cholesterol. N. Engl. J. Med. 2004, 350, 1505-1515.

7. Bell, K.J.L.; Irwig, L.; Craig, J.C.; Macaskill, P. Use of randomised trials to decide when to monitor response to new treatment. BMJ 2008, 336, 361-365. [CrossRef] 
8. Glasziou, P.P.; Irwig, L.; Aronson, J.K. Evidence-Based Medical Monitoring: From Principles to Practice; John Wiley \& Sons: New York, NY, USA, 2008.

9. National Cholesterol Education Program (NCEP) Expert Panel on Detection, Evaluation, and Treatment of High Blood Cholesterol in Adults. Third Report of the National Cholesterol Education Program (NCEP) Expert Panel on Detection, Evaluation, and Treatment of High Blood Cholesterol in Adults (Adult Treatment Panel III) Final Report. Circulation 2002, 106, 3143. [CrossRef]

10. Jellinger, P.S.; Handelsman, Y.; Rosenblit, P.D.; Bloomgarden, Z.T.; Fonseca, V.A.; Garber, A.J.; Grunberger, G.; Guerin, C.K.; Bell, D.S.H.; Mechanick, J.I.; et al. American Association of Clinical Endocrinologists and American College of Endocrinology guidelines for management of dyslipidemia and prevention of cardiovascular disease. Endocr. Pract. 2017, 23, 1-87. [CrossRef]

11. Stone, N.J.; Robinson, J.G.; Lichtenstein, A.H.; Noel Bairey Merz, C.; Blum, C.B.; Eckel, R.H.; Goldberg, A.C.; Gordon, D.; Levy, D.; Lloyd-Jones, D.M.; et al. 2013 ACC/AHA guideline on the treatment of blood cholesterol to reduce atherosclerotic cardiovascular risk in adults: A report of the American College of Cardiology/American Heart Association Task Force on Practice Guidelines. J. Am. Coll. Cardiol. 2014, 63, 2889-2934. [CrossRef]

12. GBD 2015 Neurological Disorders Collaborator Group. Global, regional, and national burden of neurological disorders during 1990-2015: A systematic analysis for the Global Burden of Disease Study 2015. Lancet Neurol. 2017, 16, 877-897. [CrossRef]

13. Cheol Seong, S.; Kim, Y.Y.; Khang, Y.H.; Heon Park, J.; Kang, H.J.; Lee, H.; Do, C.H.; Song, J.S.; Hyon Bang, J.; Ha, S.; et al. Data resource profile: The national health information database of the National Health Insurance Service in South Korea. Int. J. Epidemiol. 2016, 46, 799-800. [CrossRef] [PubMed]

14. Seong, S.C.; Kim, Y.-Y.; Park, S.K.; Khang, Y.H.; Kim, H.C.; Park, J.H.; Kang, H.-J.; Do, C.-H.; Song, J.-S.; Lee, E.-J.; et al. Cohort profile: The National Health Insurance Service-National Health Screening Cohort (NHIS-HEALS) in Korea. BMJ Open 2017, 7, e016640. [CrossRef] [PubMed]

15. Mozaffarian, D.; Benjamin, E.J.; Go, A.S.; Arnett, D.K.; Blaha, M.J.; Cushman, M.; Das, S.R.; de Ferranti, S.; Després, J.P.; Fullerton, H.J.; et al. Heart disease and stroke statistics-2016 update: A report from the American Heart Association. Circulation 2016, 133, e38-e48. [CrossRef]

16. Peterson, A.M.; Nau, D.P.; Cramer, J.A.; Benner, J.; Gwadry-Sridhar, F.; Nichol, M. A checklist for medication compliance and persistence studies using retrospective databases. Value Health 2007, 10, 3-12. [CrossRef] [PubMed]

17. Bell, K.J.L.; Kirby, A.; Hayen, A.; Irwig, L.; Glasziou, P. Monitoring adherence to drug treatment by using change in cholesterol concentration: Secondary analysis of trial data. BMJ 2011, 342, d12. [CrossRef]

18. Long-Term Intervention with Pravastatin in Ischaemic Disease (LIPID) Study Group. Prevention of cardiovascular events and death with pravastatin in patients with coronary heart disease and a broad range of initial cholesterol levels. N. Engl. J. Med. 1998, 339, 1349-1357. [CrossRef] [PubMed]

19. Tonkin, A.; Barter, P.; Best, J.; Boyden, A.; Furler, J.; Hossack, K.; Sullivan, D.; Thompson, P.; Vale, M.; Cooper, C.; et al. National Heart Foundation of Australia and the Cardiac Society of Australia and New Zealand: Position statement on lipid management-2005. Heart Lung Circ. 2005, 14, 275-291. [PubMed]

20. National Institute for Health and Clinical Excellence. Lipid Modification-Primary and Secondary CVD Prevention-Management. What Follow Up Is Recommended after Initiation of Statin Therapy? National Institute for Health and Care Excellence: London, UK, 2009.

21. Rachmani, R.; Slavachevski, I.; Avin, M.; Levi, Z.; Ravid, M. Teaching patients to monitor their risk factors retards the progression of vascular complications in high-risk patients with Type 2 diabetes mellitus-a randomized prospective study. Diabet. Med. 2002, 19, 385-392. [CrossRef] [PubMed]

22. Benner, J.S.; Tierce, J.C.; Ballantyne, C.M.; Prasad, C.; Bullano, M.F.; Willey, V.J.; Erbey, J.; Sugano, D.S. Follow-up lipid tests and physician visits are associated with improved adherence to statin therapy. PharmacoEconomics 2004, 22, 13-23. [CrossRef] [PubMed]

23. Brookhart, M.A.; Patrick, A.R.; Schneeweiss, S.; Avorn, J.; Dormuth, C.; Shrank, W.; van Wijk, B.L.; Cadarette, S.M.; Canning, C.F.; Solomon, D.H. Physician follow-up and provider continuity are associated with long-term medication adherence: A study of the dynamics of statin use. Arch. Intern. Med. 2007, 167, 847-852. [CrossRef] [PubMed]

24. Maeda, K.; Noguchi, Y.; Fukui, T. The effects of cessation from cigarette smoking on the lipid and lipoprotein profiles: A meta-analysis. Prev. Med. 2003, 37, 283-290. [CrossRef] 
25. Rabar, S.; Harker, M.; O'Flynn, N.; Wierzbicki, A.S.; on behalf of the Guideline Development Group. Lipid modification and cardiovascular risk assessment for the primary and secondary prevention of cardiovascular disease: Summary of updated NICE guidance. BMJ 2014, 349, g4356. [CrossRef] [PubMed]

26. Ferket, B.S.; Colkesen, E.B.; Visser, J.J.; Spronk, S.; Kraaijenhagen, R.A.; Steyerberg, E.W.; Hunink, M.M. Systematic review of guidelines on cardiovascular risk assessment: Which recommendations should clinicians follow for a cardiovascular health check? Arch. Intern. Med. 2010, 170, 27-40. [CrossRef] [PubMed]

27. Robertson, I.; Phillips, A.; Mant, D.; Thorogood, M.; Fowler, G.; Fuller, A.; Yudkin, P.; Woods, M. Motivational effect of cholesterol measurement in general practice health checks. Br. J. Gen. Pract. 1992, 42, 469-472.

28. Guerci, B.; Drouin, P.; Grangé, V.; Bougnères, P.; Fontaine, P.; Kerlan, V.; Passa, P.; Thivolet, C.; Vialettes, B.; Charbonnel, B. Self-monitoring of blood glucose significantly improves metabolic control in patients with type 2 diabetes mellitus: The Auto-Surveillance Intervention Active (ASIA) study. Diabetes Metab. 2003, 29, 587-594. [CrossRef]

29. Green, B.B.; Cook, A.J.; Ralston, J.D.; Fishman, P.A.; Catz, S.L.; Carlson, J.; Carrell, D.; Tyll, L.; Larson, E.B.; Thompson, R.S. Effectiveness of home blood pressure monitoring, Web communication, and pharmacist care on hypertension control: A randomized controlled trial. JAMA 2008, 299, 2857-2867. [CrossRef]

30. Shepherd, J.; Barter, P.; Carmena, R.; Deedwania, P.; Fruchart, J.-C.; Haffner, S.; Hsia, J.; Breazna, A.; LaRosa, J.; Grundy, S.; et al. Effect of Lowering LDL Cholesterol Substantially Below Currently Recommended Levels in Patients With Coronary Heart Disease and Diabetes: The Treating to New Targets (TNT) study. Diabetes Care 2006, 29, 1220-1226. [CrossRef]

31. Colhoun, H.M.; Betteridge, D.J.; Durrington, P.N.; Hitman, G.A.; Neil, H.A.; Livingstone, S.J.; Thomason, M.J.; Mackness, M.I.; Charlton-Menys, V.; Fuller, J.H.; et al. Primary prevention of cardiovascular disease with atorvastatin in type 2 diabetes in the Collaborative Atorvastatin Diabetes Study (CARDS): Multicentre randomised placebo-controlled trial. Lancet 2004, 364, 685-696. [CrossRef]

32. Lindenstrom, E.; Boysen, G.; Nyboe, J. Influence of total cholesterol, high density lipoprotein cholesterol, and triglycerides on risk of cerebrovascular disease: The Copenhagen city heart study. BMJ 1994, 309, 11-15. [CrossRef]

33. Iso, H.; Jacobs, D.R., Jr.; Wentworth, D.; Neaton, J.D.; Cohen, J.D.; MRFIT Research Group. Serum cholesterol levels and six-year mortality from stroke in 350,977 men screened for the multiple risk factor intervention trial. N. Engl. J. Med. 1989, 320, 904-910. [CrossRef]

34. Vergouwen, M.D.; De Haan, R.J.; Vermeulen, M.; Roos, Y.B. Statin Treatment and the Occurrence of Hemorrhagic Stroke in Patients With a History of Cerebrovascular Disease. Stroke 2008, 39, 497-502. [CrossRef]

35. Park, B.; Sung, J.; Park, K.; Seo, S.; Kim, S. Strategies to Improve the Validity of Diagnostic Codes of National Health Insurance Claims Data; Health Insurance Review and Assessment Services: Seoul, Korea, 2002; pp. 118-119.

36. Kim, J. Strategies to Enhance the Use of National Health Insurance Claims Database in Generating Health Statistics; Health Insurance Review and Assessment Services: Seoul, Korea, 2005.

37. Mihaylova, B.; Emberson, J.; Blackwell, L.; Keech, A.; Simes, J.; Barnes, E.H.; Voysey, M.; Gray, A.; Collins, R.; Baigent, C. The effects of lowering LDL cholesterol with statin therapy in people at low risk of vascular disease: Meta-analysis of individual data from 27 randomised trials. Lancet 2012, 380, 581-590. [PubMed]

38. Hokanson, J.E.; Austin, M.A. Plasma Triglyceride Level is a Risk Factor for Cardiovascular Disease Independent of High-Density Lipoprotein Cholesterol Level: A Metaanalysis of Population-Based Prospective Studies. Eur. J. Cardiovasc. Risk 1996, 3, 213-219. [CrossRef]

39. Heiss, G.; Leon, A.; Pekkanen, J.; Linn, S.; Suchindran, C.M.; Rifkind, B.M.; Tyroler, H.A. Ten-Year Mortality from Cardiovascular Disease in Relation to Cholesterol Level among Men with and without Preexisting Cardiovascular Disease. N. Engl. J. Med. 1990, 322, 1700-1707.

40. Gordon, D.J.; Probstfield, J.L.; Garrison, R.J.; Neaton, J.D.; Castelli, W.P.; Knoke, J.D.; Jacobs, D.R., Jr.; Bangdiwala, S.; Tyroler, H.A. High-density lipoprotein cholesterol and cardiovascular disease. Four prospective American studies. Circulation 1989, 79, 8-15. [CrossRef]

41. Fryer, A.A.; Smellie, W.S. Managing demand for laboratory tests: A laboratory toolkit. J. Clin. Pathol. 2013, 66, 62-72. [CrossRef]

(C) 2019 by the authors. Licensee MDPI, Basel, Switzerland. This article is an open access article distributed under the terms and conditions of the Creative Commons Attribution (CC BY) license (http://creativecommons.org/licenses/by/4.0/). 